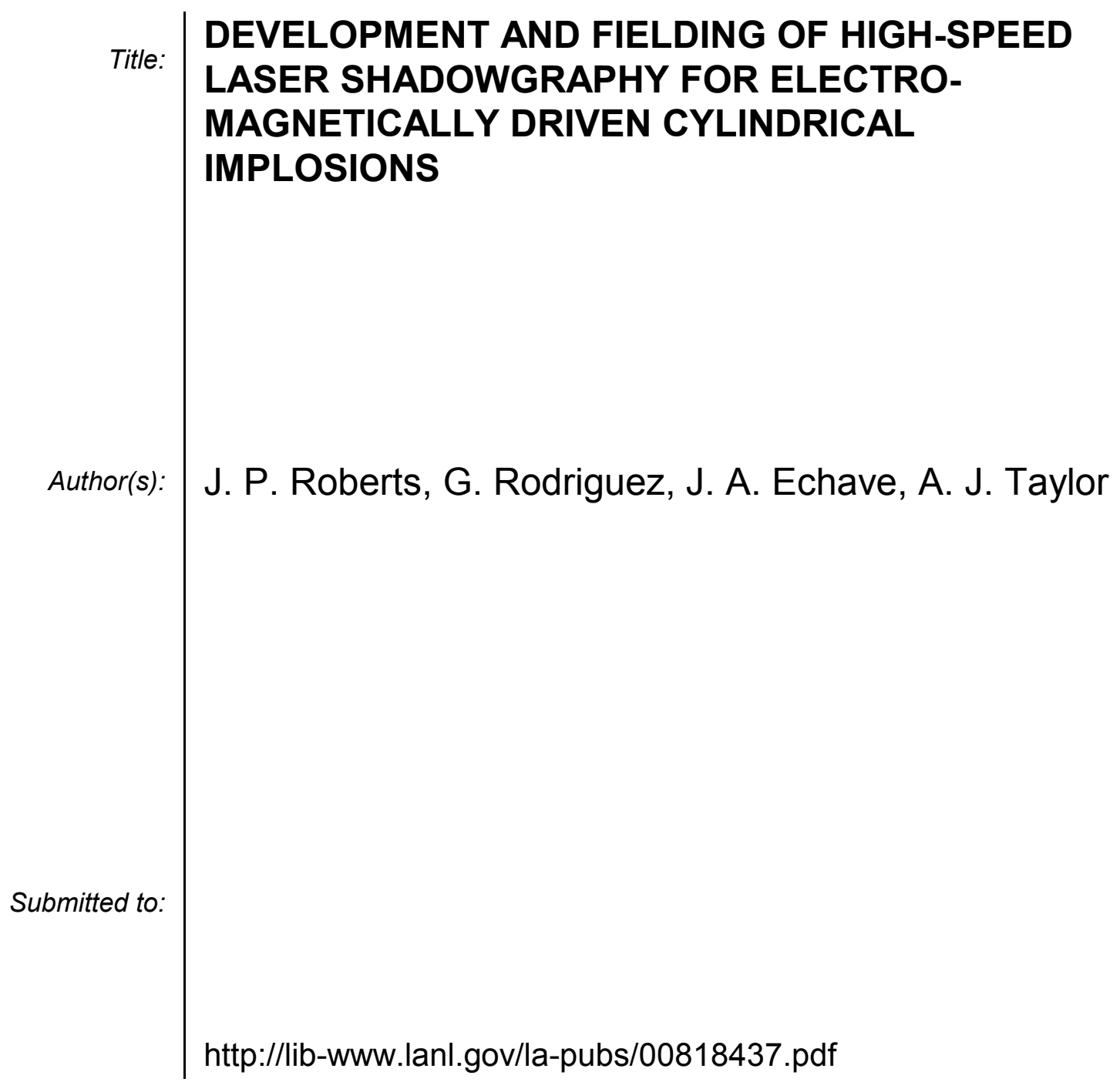




\title{
DEVELOPMENT AND FIELDING OF HIGH-SPEED LASER SHADOWGRAPHY FOR ELECTRO-MAGNETICALLY DRIVEN CYLINDRICAL IMPLOSIONS
}

\author{
J. P. Roberts, G. Rodriguez", J. A. Echave, A. J. Taylor \\ Los Alamos National Laboratory, MS K764 \\ Los Alamos, NM 87545 USA
}

\section{Abstract}

A laser shadowgraphy system for high-speed imaging of a convergent cylindrical shockwave generated by an electro-magnetically driven solid density liner implosion in Lucite is described. The laser shadowgraphy system utilizes an advanced high-energy, long-pulse, frequencydoubled Nd:YAG laser for target illumination and a fast framing camera for multiple frame imaging of the shockwave as it radially converges and transits the Lucite. The time window resolution is $10 \mathrm{~ns}$ as determined by the fastest exposure time capable with the camera. Two onaxis symmetric implosions and two off-axis antisymmetric implosion experiments were fielded at the Air Force Research Laboratory's Shiva Star 4.2 MJ capacitor bank $z$-pinch facility. For each experimental shot, the shadowgraphy system captured several frames of shadowgraph images as the shockwave moved through the Lucite. Analysis of the shockwave shadowgraph image shapes is done by fitting each shadowgraph image to a generic elliptical fit function and plotting the resultant 2-D image fits for comparison. For the on-axis symmetric implosion shots, a radial shock velocity is calculated. The Lucite shock speed is seen to increase monotonically from an initial velocity of $7.9 \mathrm{~mm} / \mu \mathrm{s}$ to a near final velocity of $13.4 \mathrm{~mm} / \mu \mathrm{s}$ as convergence effects dominate the shock speed calculated at small radii.

\section{INTRODUCTION}

Experimental characterization of a converging shockwave in a cylindrical geometry provides fundamental benchmarks used in the modeling of 1-D and 2-D hydrodynamic phenomena from high or solid density implosions [1,2]. A high-speed laser shadowgraphy system for detailed imaging of a convergent cylindrical shockwave provides diagnosis where multiple sampling of the shock front position and radial shape are desired. We present the development of an advanced laser shadowgraphy system: a novel laser source, the fiber optic delivery system, double-pass target imaging, and fast frame camera image acquisition, recently fielded on a set of hydrodynamic implosion experiments. While laser and flashlamp shadowgraphy have been utilized for a number of years [3], the requirements of these experiments necessitated the development of unique powerful visible laser light source with the capability of constant target illumination for periods of up to $50 \mu \mathrm{s}$.

Labeled the Near Term Liner Experiments (NTLX), an experimental series of four shots were conducted at the Air Force Research Laboratory's Shiva Star 1300- $\mu \mathrm{f}$ capacitor bank $z$-pinch facility $[2,4]$ in Albuquerque, NM for the High Energy Density Hydrodynamics Program at the Los Alamos National Laboratory. Since additional details on the Shiva Star NTLX cylindrical liner implosion experimental series can be found in other papers from these conference proceedings $[4,5,6]$, we describe only brief details of the NTLX experimental series performance parameters. The configuration geometry for these experiments is shown in Figure 1. The 1100-aluminum liner acceleration is initiated by discharging the $1300 \mu \mathrm{f}$ Shiva Star capacitor bank at an $80 \mathrm{kV}$ charging voltage. The discharge current travels along the $40-\mathrm{mm}$ length of the liner and rises to $16 \mathrm{MA}$ in approximately $8 \mu \mathrm{s}$. Initial inner radius and thickness of the liner are $50 \mathrm{~mm}$ and 1 $\mathrm{mm}$, respectively. Azimuthal magnetic fields on the liner surfaces exceed 0.5 MG. The liner is accelerated to $\sim 6.2$ $\mathrm{km} / \mathrm{s}$ and is driven symmetrically onto a concentric

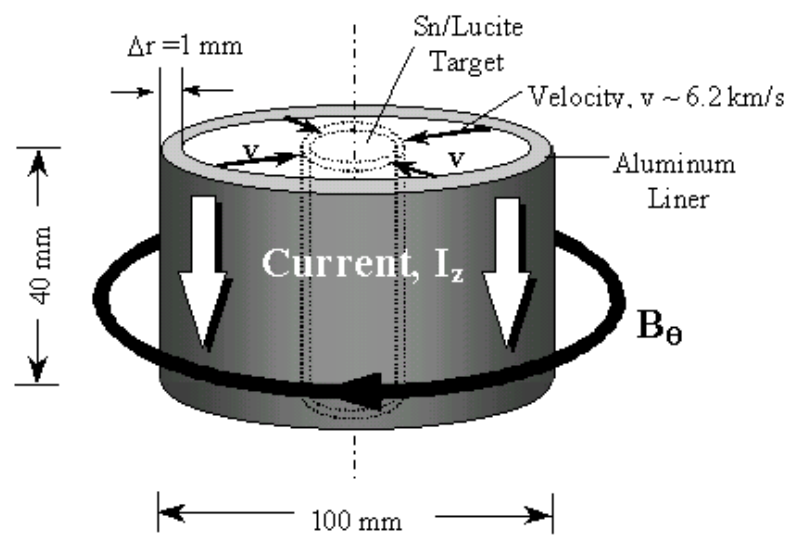

Figure 1. A simple diagram of a cylindrical z-pinch liner-target implosion experimental geometry. Approximately $16 \mathrm{MA}$ of current, $\mathrm{I}_{\mathrm{z}}$, from the Shiva Star capacitor bank is sent axially along the $\mathrm{Al}$ liner walls, and an azimuthal magnetic field, $B_{\theta}$, is setup. The liner is accelerated inward toward the target for implosion. The liner impacts the $\mathrm{Sn} /$ Lucite target at an approximate velocity of $6.2 \mathrm{~km} / \mathrm{s}$ to setup the convergent shockwave. 
$\mathrm{Sn} /$ Lucite target, and a convergent shockwave is setup in the 15 -mm radius Lucite rod. The liner-target collision time occurs at $\sim 13.7 \mu \mathrm{s}$. After the liner-target collision, a shockwave is setup in the outer tin target shell, and the shockwave begins to propagate radially inward toward the center Lucite post. The initial Lucite radius is $15 \mathrm{~mm}$, and the transit of the shockwave through the Lucite is imaged by the shadowgraphy system. Both, on-axis and off-axis, concentric Lucite targets are used to study symmetric and asymmetric shockwave motion. The shockwave in the Lucite is recorded by multiple image sampling with the shadowgraphy system. The main feature of our shadowgraphy system consists of a unique frequencydoubled long-pulse Nd:YAG laser and fast framing camera capability that allows us to capture multiple images of the shock transit through the Lucite. We briefly describe the details of our shadowgraphy system and present the experimental results of our measurements.

\section{SHADOWGRAPHY SYSTEM}

Experimental details of our laser shadowgraphy system are given elsewhere [7], so we only give a brief desciprion here. Pulses from a custom built, single-frequency, longpulse frequency-doubled $\mathrm{Nd}: \mathrm{YAG}$ laser at $532 \mathrm{~nm}$ are launched along a $550 \mu \mathrm{m}$ large core fiber for beam delivery to the Shiva Star target chamber. The Nd:YAG laser allows for high output energy $(75 \mathrm{~mJ})$ with temporally adjustable (100 ns to $50 \mu \mathrm{s})$ square pulses. A simple schematic of our fiber optic transport and image relay system is shown in Figure 2. Pulses from the shadowgraphy laser system are fiber-optic coupled and launched into a $50-\mathrm{m}$ long $550-\mu \mathrm{m}$ core fused silica optical fiber. This allows for the laser system to be remotely located free from potential EMI transients produced upon firing the Shiva Star capacitor bank. Fiber coupling of only $5 \mathrm{~mJ}$ in a $10 \mu$ s laser pulse $(500 \mathrm{~W})$ is

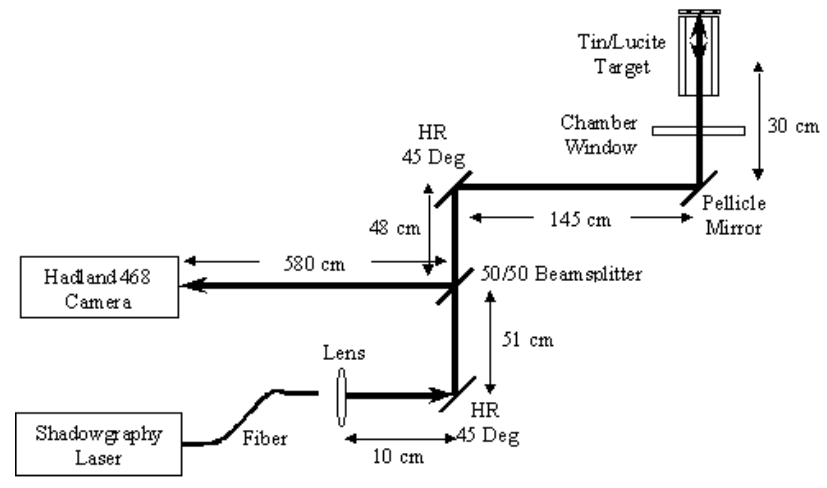

Figure 2. Laser shadowgraphy system optical path layout. Approximate distance from the target to camera is 8 meters. Isolation of the laser from the Shiva Star bank noise is accomplished by fiber-coupling the laser output to the experiment via a 50 meter $550-\mu \mathrm{m}$ core optical fiber. more than adequate to obtain good camera image signals. After fiber optic beam transport, a single plano-convex 4inch diameter $\mathrm{f}=15 \mathrm{~cm}$ BK7 lens is used to collimate the beam though the steering optics, target, and camera. The collimated $35-\mathrm{mm}$ diameter beam is periscoped and steered to the target before being doubled-passed through the target and sent over to the recording camera using a 2inch diameter 50/50 dielectric 532-nm beamsplitter. Although not optimal, double-pass illumination of the target was necessary in order to accommodate additional hardware for axial imaging of the shockwave with x-ray radiography. Double-pass transport of the beam through the $30-\mathrm{mm}$ diameter Lucite target is accomplished by evaporating a $50 \AA$ and $2500 \AA$ thick layer of titanium and aluminum on the top of the highly polished end face of the Lucite. This evaporated layer of aluminum serves to reflect the laser beam back down through the Lucite to the camera for recording.

High-speed electronic image recording is accomplished with a Hadland 468 fast-framing camera located approximately 8 meters away from the target. The Hadland 468 camera is setup to acquire eight frames at 10 ns exposure per frame. Prisimatic splitting of the image to eight independent microchannel plate image intensifiedCCD (ICCD) modules allows for independent enhancement of each frame. Each ICCD module is $586(\mathrm{H})$ pixels $\mathrm{x} 385(\mathrm{~W})$ pixels with $22 \mu \mathrm{m}$ per pixel, ultimately producing a composite image 2344(H) x $770(\mathrm{~W})$ pixels for all eight frames. The framing camera input optics includes a 3.5-inch diameter aperture Questar telescope lens, $\mathrm{f}=1600 \mathrm{~mm}$ and $\mathrm{f}$-number of 16 . For all four NTLX experiments, static and dynamic event shadowgraph images are captured and stored.

Electronic timing of the entire shadowgraphy system to the Shiva Star capacitor bank is accomplished using a coincidence circuit between the laser and the capacitor bank trigger circuitry. In order to fully capture the shockwave implosion in the Lucite, the shadowgraphy laser turn-on time and pulse width are set to $10.0 \mu$ s and $8.5 \mu \mathrm{s}$, respectively. All camera image acquisition framing times are referenced to machine current start and are typically between $13.0-16.3 \mu \mathrm{s}$.

\section{RESULTS AND DISCUSSION}

Static visible photographs of the tin/Lucite target assembly are shown in Figure 3 for (a) the symmetric on axis concentric case and the (b) asymmetric off-axis case. In all cases the Lucite diameter is kept fixed at $30 \mathrm{~mm}$, and the tin outer diameter is kept fixed at $40 \mathrm{~mm}$. For the on-axis case, the tin wall thickness and overall diameter is $5 \mathrm{~mm}$ and $40 \mathrm{~mm}$, respectively. For the off-axis case, only the Lucite is radially offset by $4 \mathrm{~mm}$. This forces a tin minimum and maximum wall thickness of $1 \mathrm{~mm}$ and 9 $\mathrm{mm}$, respectively. The NTLX experimental series consisted of four shots with NTLX1 and NTLX3 having on-axis target assembly geometries and NTLX2 and 

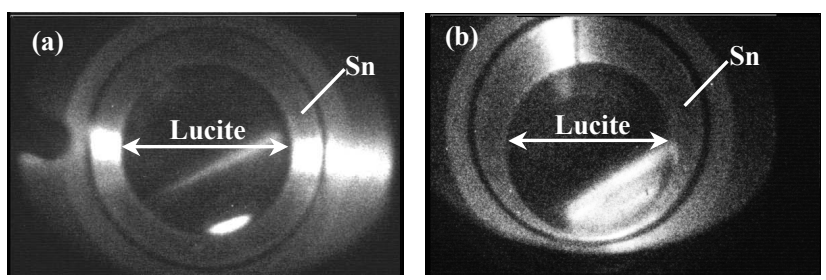

Figure 3. Visible photograph end view images of the two geometries used in the NTLX experiments: (a) symmetric on-axis concentric case, and (b) anti-symmetric off-axis case.

NTLX4 having off-axis target assembly geometries. In Figure 4 we show the dynamic shadowgraph images for (a) NTLX3 (on-axis) and (b) NTLX4 (off-axis) cases with the framing times as indicated in the figure. The differences between symmetric and asymmetric shockwave propagation is clearly distinguishable between NTLX3 and NTLX4. That is, in the asymmetric case, the shockwave begins to first emerge from the tin/Lucite boundary where the tin wall is at a minimum, $1 \mathrm{~mm}$ along the offset axis. Furthermore, once the shockwave is fully contained in the Lucite, the shockwave shape is somewhat elliptical and does not converge to initial 4-mm center offset point for the asymmetric case. This is principally due to unequal shock propagation caused by the shock emerging from the thin side of the tin wall before the thick side of the tin wall. This observation is revealed in the analysis of the late time frame shadowgraph images as described below. By contrast, for the symmetric on-axis shockwave propagation case, late time frame shadowgraph image analysis shows that the shockwave convergence center point is quite close to the initial on-axis center position.

In the double pass shadowgraphy arrangement described here, comparison of the shadowgraph images to the visible

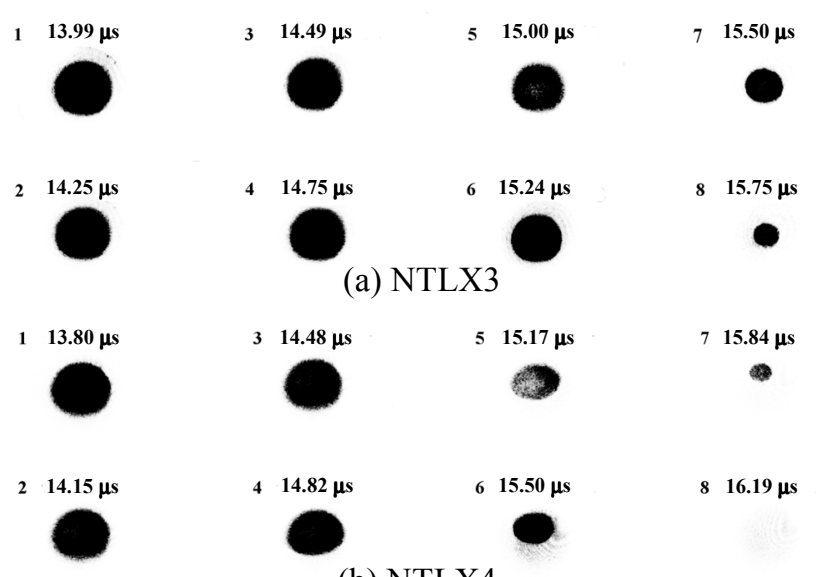

(b) NTLX4

Figure 4. Sets of dynamic laser shadowgraph images captured by the eight-frame fast framing camera for the (a) NTLX3 (on-axis) and (b) NTLX4 (off-axis) shots. The exposure time for each frame is $10 \mathrm{~ns}$, and the interframe time is $250 \mathrm{~ns}$ for NTLX3 and $340 \mathrm{~ns}$ for NTLX4. All frame times are referenced to Shiva Star machine current start static images is not possible because of the perspective or view angle distortion due to non-telecentric lens imaging. Instead, image analysis is achieved by comparing the dynamic shadowgraphs with the static shadowgraphs in order to measure the shockwave position and shape. Two dimensional image analysis of the shockwave shape is accomplished by fitting all of the shadowgraph images for each frame to the generic elliptic function, $\left(\left(x-x_{o}\right) / a\right)^{2}+\left(\left(y-y_{o}\right) / b\right)^{2}=1$, where the major and minor axes lengths are $2 a$ and $2 b$, respectively. The fits to the edges of the shadowgraph images are done for the static and the dynamic data for all the experimental shots (NTLX 1-4). Any comparison between actual experimental physical dimensions must be done on a frame-by-frame basis in which the static shadowgraph for each individual frame is used as the reference normalization for the dynamic shadowgraph. The unshocked static shadowgraph image is assumed to have a initial Lucite diameter of $15 \mathrm{~mm}$. With this in mind, we plot the 2-D shadowgraph image fits in Figure 5 and comment on our results. In Figure 5(a) for NTLX1, an on-axis symmetric impact shot, only three frames of dynamic data were acquired due to a timing error in the predicted shock arrival time resulting in late image capture of the shock transit in the Lucite. The results for NTLX1 clearly show a shock convergent on center to approximately $0.6 \mathrm{~mm}$ at $15.79 \mu \mathrm{s}$. At $\mathrm{t}=15.79 \mu \mathrm{s}$, the unshocked Lucite diameter is approximately $17 \%$ of its initial static diameter of $30 \mathrm{~mm}$. Figure 5(b) shows the shadowgraph image fits for the second on-axis symmetric shot, NTLX3. Three frames of dynamic shadowgraphs (Frames 6 - 8) capture the shockwave transit in the Lucite. For $\mathrm{t} \leq 15.00 \mu \mathrm{s}$ (Frames 1-5), the shockwave has not

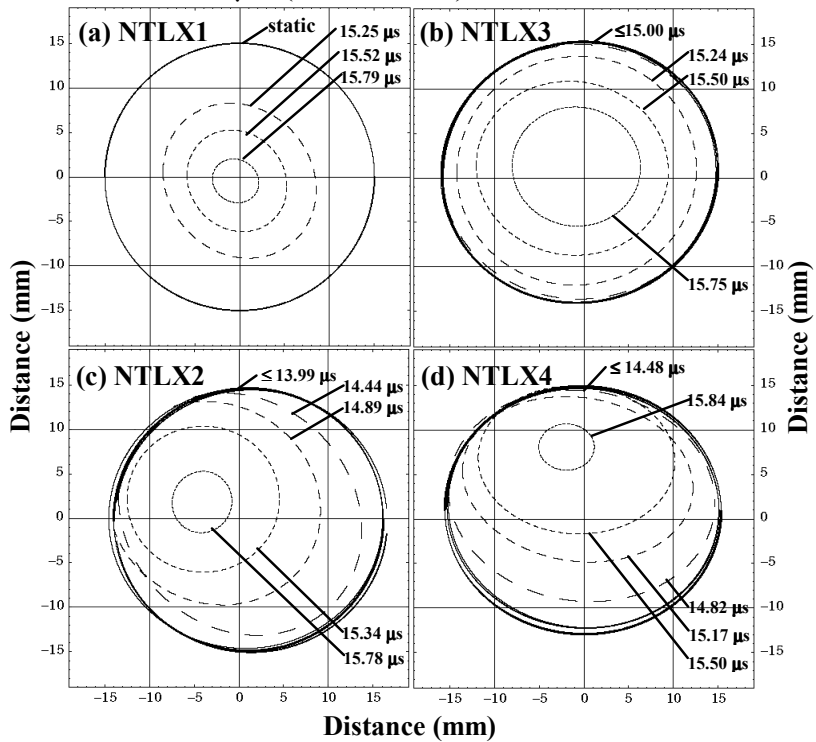

Figure 5. Ellispe fitted 2-D plots of the shockwave images in Lucite for (a) NTLX1, on-axis; (b) NTLX3, onaxis; (c) NTLX2, off-axis; and (d) NTLX4, off-axis shots for the given frame capture times as marked. Clear differentiation between on-axis and off-axis shots is observed. 
emerged from the Sn/Lucite interface. At $\mathrm{t}=15.24 \mu \mathrm{s}$, the shockwave front appears in the Lucite at a diameter of $26.2 \mathrm{~mm}$. As in NTLX1, excellent shock convergence to center is measured and is to approximately $1.5 \mathrm{~mm}$ of the original static center position. At $\mathrm{t}=15.75 \mu \mathrm{s}$, the unshocked Lucite diameter is $46 \%$ of its initial static diameter. Figures 5(c) and 5(d) show the shadowgraph image fit results for the off-axis anti-symmetric impact shots NTLX2 and NTLX4, respectively. The Lucite offset from center is $4 \mathrm{~mm}$, forcing the tin wall to vary from a maximum of $9 \mathrm{~mm}$ to a minimum of $1 \mathrm{~mm}$ along the offset direction (see Figure 3(b)). For NTLX2 and NTLX4, four frames of dynamic shadowgraphs (Frames 3-7) for each shot capture the transit of the shockwave in the Lucite. For the shadowgraph images closest to convergence, the NTLX2 (Frame 7) results show that at $15.78 \mu$ s the unshocked Lucite is at $23 \%$ of its initial 30 $\mathrm{mm}$ diameter. Calculation of the center point for this frame also shows that there is a shift of approximately 4.5 $\mathrm{mm}$ between the static center position and the dynamic center position. As mentioned above, offset shock convergence is expected from the static center because of asymmetric emergence of the shockwave from the thin tin wall side into the Lucite before emerging from the thick side of the tin wall. Similarly for NTLX4 in Figure 5(d), the unshocked Lucite is at $38 \%$ of its initial diameter at $\mathrm{t}=$ $15.84 \mu \mathrm{s}$ for Frame 7, and the dynamic center point is also offset by approximately $8.4 \mathrm{~mm}$ from the static center position. It is stressed here that shot-to-shot variation of the liner-target impact and shock convergence times in the Lucite are extremely sensitive to the aluminum liner acceleration profile and the exact Shiva Star machine current drive parameters. Although the nominal Shiva Star capacitor bank charge voltage was $80 \mathrm{kV}$, changes of only a few percent in the peak current can cause several hundreds of nanoseconds shifts in the impact and convergence times. Therefore, any detailed shot-to-shot comparison of the data requires a full analysis of the action of the current on liner acceleration and liner-target hydrodynamics [2].

From the NTLX1 and NTLX3 data in Figure 5, an average radial shock velocity is calculated by taking the average radius of each elliptic fit from both of these experimental shots. The Lucite shock speed is seen to increase monotonically from an initial velocity of 7.9 $\mathrm{mm} / \mu \mathrm{s}$ to a near final velocity of $13.4 \mathrm{~mm} / \mu \mathrm{s}$ as convergence effects dominate the shock speed calculated at small radii.

The results presented above demonstrate that our laser shadowgraphy system is capable of capturing images of the propagation and shapes of cylindrically convergent shockwaves in Lucite at shock speeds exceeding 10 $\mathrm{mm} / \mu \mathrm{s}$. Although Lucite was an excellent candidate for shadowgraph imaging of the shockwave, future experiments in other transparent solids or liquids may preclude us from using the shadowgraph technique in cases where the material is not expected to become optically opaque under shock loading, such as in weak shocks that only slightly modify the material optical index. In such cases, the complementary technique of laser Schlieren imaging may be more appropriate since the Schlieren method is directly sensitive to changes in the density gradient of the material. Nonetheless, the shadowgraphy results presented here provide useful 2-D shockwave imaging information for magnetohydrodynamic computer analysis of materials under dynamic shock loading from electro-magnetically driven cylindrical implosions.

This work was supported by University of California for the High Energy Density and Hydrodynamics Program at the Los Alamos National Laboratory under the auspices of the Department of Energy contract number W-7405-ENG36.

\section{REFERENCES}

[1] R. L. Bowers, J. H. Brownell, H. Lee, K. D. McLenithan, A. J. Scannapieco, and W. R. Shanahan, "Design and modeling of precision solid liner experiments on Pegasus," J. Appl. Phys., vol. 83, pp.4146-4159, Apr. 1998.

[2] R. Kanzleiter,"Using pulsed power for hydrodynamic code verification and validation," paper\# P3-D12 these proceedings.

[3] F. Amiranoff, R. Fedosejevs, R. F. Schmalz, R. Sigel, and Y. Teng,"Laser driven shock wave studies using optical shadowgraphy," Phys. Rev. A, vol. 32, pp. 35353546, Dec. 1985.

[4] P. J. Turchi et al.,"Design and operation of high energy density liner implosions at 16 MA for studies of converging shocks," paper\# O3B5 these proceedings.

[5] W. Anderson et al.,"A proposed Atlas liner design fabricated for hydrodynamic experiments on Shiva Star," paper\# P3-D15 these proceedings.

[6] R. T. Olson, D. M. Oro, B. G. Anderson, and J. K. Studebaker,"Radiographic results from the NTLX series of hydrodynamic experiments," paper\# O3B8 these proceedings.

[7] G. Rodriguez, J. P. Roberts, J. A. Echave, and A. J. Taylor, "High speed laser shadowgraphy for electromagnetically driven cylindrical implosions," Rev. Sci. Instrum., vol.72, Aug. 2001, in press. 